\title{
First numerical ephemerides of the Martian moons
}

\author{
V. Lainey ${ }^{1,2}$, V. Dehant ${ }^{1}$, and M. Pätzold ${ }^{3}$ \\ 1 Royal Observatory of Belgium, 3 avenue Circulaire, 1180 Bruxelles, Belgium \\ e-mail: Lainey@oma.be \\ 2 Institut de Mécanique Céleste et de Calcul des Ephémérides-Observatoire de Paris, UMR 8028 du CNRS, \\ 77 avenue Denfert-Rochereau, 75014 Paris, France \\ 3 Institut für Geophysik und Meteorologie, Universität zu Köln, Albertus-Magnus-Platz, 50923 Cologne, Germany
}

Received 20 April 2006 / Accepted 22 December 2006

\begin{abstract}
We present new ephemerides of Phobos and Deimos that are fit to observations from 1877 to 2005 and include recent spacecraft observations by Mars Global Surveyor and Mars Express. In contrast to earlier models, this is the first completely numerical one. In particular, the tidal effects have been modeled by the tidal bulge raised by each moon on the planet, instead of fitting secular accelerations in the satellite longitudes. This partly avoids absorbing the Deimos observational errors in its related tidal acceleration. Moreover, applying this model to other systems will be easier. Our estimate of the Martian dissipation is $Q=79.91 \pm 0.69$ ( $1 \sigma$-formal error) when assuming $k_{2}=0.152$ for the Martian Love number and $G m_{\mathrm{Ph}}=0.68 \times 10^{6} \mathrm{~m}^{3} / \mathrm{s}^{2}$ for the Phobos mass. We also report the possibility of fitting the Phobos oblateness gravity field. We suspect a non-uniform density for Phobos or a bias in either the observations or the Martian gravity field. A FORTRAN subroutine that computes the Martian moons' ephemerides is available on request.
\end{abstract}

Key words. planets and satellites: general - ephemerides - celestial mechanics - astrometry

\section{Introduction}

The orbital motions of the Martian moons are among those most studied in the Solar System. Since their discovery in 1877, a variety of dynamical models, first fit to Earth-based observations and then to spacecraft observations, have been developed. Ephemerides have been developed by Sinclair (1972), Shor (1975), and Sinclair (1978) during the Viking era, confirming the secular tidal acceleration previously found by Sharpless (1945). Later, in the early 1990's, more orbital studies were developed mainly in support of the Phobos 2 spacecraft mission. Of particular interest were the ephemerides derived by Jacobson et al. (1989) and by Morley (1990) that were used by JPL and ESOC, respectively. These ephemerides are available in the SPICE library at the address ftp://naif.jpl .nasa.gov/pub/naif/MEX/kernels/spk.

No new adjustments to the observations were done before 2005. As a result, the Martian moon ephemerides have drifted, as pointed out by Neumann et al. (2004), who used the passive mode of the MOLA instrument onboard Mars Global Surveyor (MGS) to detect the Phobos shadow on the Martian surface. After data reduction, they found that the observed position of Phobos was ahead of its predicted position by a difference of $6 \mathrm{~s}$ of time $(12 \mathrm{~km})$. This was later confirmed by Bell et al. (2005), using observations from the Mars Exploration rovers (MER). With some 6 transit events (including two events by Deimos), they also found a significant drift of $38 \mathrm{~km}$ on the Deimos position.

Bills et al. (2005) used some 16 observations covering the years 1998-2004 to correct the shift between the observed and computed positions of Phobos. They used the model of Jacobson et al. (1989) and fit only three terms from the longitude expression to the MGS observations. They succeeded in reducing the former drift on the Phobos orbital motion. However, as only Phobos was observed by MOLA (the shadow of Deimos on the Martian surface being too faint), no attempt could be made to improve the motion of Deimos. Moreover, the physical parameters from 1989 are still being used. Of particular importance are the Martian gravity field and the precession, which have been significantly improved in recent years (Lemoine et al. 2001; Yuan et al. 2001; Seidelmann et al. 2002; Konopliv et al. 2006).

The Super Resolution Camera (SRC), part of the HighResolution Stereo Camera (HRSC) onboard Mars Express (MEX), started an observation campaign of the Martian moons (see Oberst et al. 2006) in 2004-2005. The analysis of the ephemerides residuals between the predictions from ESOC and JPL with a set of 36 satellite observations again confirmed the drift of the ephemerides (mainly across track for the ESOC model and along track for the JPL model). Phobos and Deimos were found $12 \mathrm{~km}$ and $50 \mathrm{~km}$, respectively, ahead of their predicted positions by the JPL model. The ESOC model predicted better with $8 \mathrm{~km}$ and $18 \mathrm{~km}$ discrepancies on the Phobos and Deimos positions, respectively.

One of the most interesting purposes for studying the orbital evolution of the Martian moons is to investigate the Martian tidal dissipation factor. Tidal dissipation induces a phase lag between the bulge direction raised by one satellite and the Mars-satellite direction. As a consequence, momentum between the planetary rotation and the satellite orbital motion is exchanged. When the satellite revolves faster than the planet rotates, a secular acceleration on the satellite longitude is generated. This effect is observed on the orbital motion of Phobos. More difficult is detecting the tidal deceleration of Deimos (this satellite revolves more 
Table 1. Catalogue of all observations used in this paper.

\begin{tabular}{|c|c|c|c|c|c|c|c|c|c|}
\hline Reference & Frame & Type & Period & Place & $\mathrm{P} / \mathrm{M}$ & $\mathrm{D} / \mathrm{M}$ & $\mathrm{P} / \mathrm{D}$ & $\mathrm{P}$ & $\mathrm{D}$ \\
\hline Morley (1989) & $1+2+3$ & $0+1+2$ & $1877-1982$ & 23 sites & 2212 & 2600 & 100 & & \\
\hline Christie et al. (1878) & 1 & 0 & $1877-1877$ & Greenwich, Oxford & 3 & 12 & & & \\
\hline Young (1880) & 1 & 0 & 1879-1879 & Princeton & 4 & 10 & & & \\
\hline Shor (1989) & $1+3+4$ & 1 & 1978-1988 & 7 sites & 268 & 221 & 195 & & \\
\hline Kiselev et al. (1989) & 1 & 1 & 1986-1986 & Ordubad & 117 & 56 & & & \\
\hline Kiseleva \& Chanturiia (1988) & 1 & 1 & 1986-1986 & Ordubad & 6 & & & & \\
\hline Bobylev et al. (1991) & 1 & 1 & $1988-1988$ & Pulkovo, Ordubad & 164 & 54 & 147 & & \\
\hline Colas (1992) & 1 & 1 & $1988-1988$ & Pic-du-Midi & & & 813 & & \\
\hline Izhakevich et al. (1990) & 4 & 1 & 1988-1988 & Majdanak & 65 & 47 & 88 & & \\
\hline Jones et al. (1989) & 1 & 0 & 1988-1988 & La Palma & 88 & 12 & 66 & & \\
\hline Kalinichenko et al. (1990) & 1 & 1 & 1988-1988 & Abastuman & 19 & 1 & 17 & & \\
\hline Nikonov et al. (1991) & 1 & 1 & 1988-1988 & Boyeros & 23 & & & & \\
\hline Tel'nyuk-Adamchuk et al. (1990) & 4 & 1 & 1988-1988 & Kiev & 99 & 74 & 32 & & \\
\hline Kudryavtsev et al. (1992) & 4 & 3 & 1988-1988 & Majdanak & & & & 660 & 639 \\
\hline Duxbury \& Callahan (1988) & 3 & 3 & 1976-1980 & Viking 1-2 & & & & 166 & 109 \\
\hline Duxbury \& Callahan (1989) & 3 & 3 & 1971-1972 & Mariner 9 & & & & 49 & 31 \\
\hline Kolyuka et al. (1991) & 3 & 3 & 1989-1989 & Phobos 2 & & & & 37 & 8 \\
\hline Pascu $(1995)^{*}$ & 1 & 1 & 1967-1988 & Flagstaff, Washington & 217 & 223 & & & \\
\hline Ledovskaya (2001)* & 4 & 3 & 1963-1988 & Kiev, Kitab, Maid. & & & & 133 & 283 \\
\hline Rohde $(2003) *$ & 1 & 0 & 2003-2003 & Flagstaff & & & 196 & & \\
\hline Bills et al. (2005) & 4 & 3 & 1977-2004 & MGS, Viking lander 1 & & & & 17 & \\
\hline Oberst et al. (2006) & 4 & 3 & 2004-2005 & Mars Express & & & & 26 & 10 \\
\hline
\end{tabular}

slowly than Mars rotates). Both accelerations, however, were fit in the former models, providing a good agreement with the Phobos acceleration. The deceleration of Deimos is still uncertain and probably more a matter of observational errors.

The density of Phobos is less constrained than the Martian tidal dissipation and usually assumed to be uniform. So far, the different flybys at Phobos by the Viking 1 and Phobos 2 spacecrafts only quantified the satellite mass. The Phobos gravity field (essentially the coefficients $C_{20}$ and $C_{22}$ ), however, induces secular terms on the mean anomaly, node, and pericenter of the Phobos motion, which are different from the quadratic behavior induced by the tidal effects. Several authors tried to use their ephemerides solution to verify the constant density assumption (see Chapront-Touze 1990; Emelyanov et al. 1993). The uncertainties on the Martian gravity field (often fit during the Martian moon ephemerides construction) were probably too large to answer this question definitively.

This paper is divided as follows. Section 2 describes all available observations of the Martian moons. Section 3 presents the numerical method introduced to model the satellite motions. Section 4 presents the fit of the model to the JPL model. In Sect. 5 the fit of our model directly to the observations is presented. Section 6 summarizes the procedure to provide the Martian moons ephemerides representation to the user. Section 7 compares the ephemerides with the JPL/ESOC ephemerides. The last section discusses the influence of few non introduced perturbations in the present model, along with the question of the density of Phobos.

\section{The observations}

The observations of the Martian moons are numerous and have different levels of accuracy. An important available database ${ }^{1}$

\footnotetext{
1 This database is available on the internet at the following addesses: http://lnfm1.sai.msu.ru/neb/nss/index.htm http://www.imcce.fr/fr/ephemerides/generateur/ saimirror/obsindhf.htm
}

was developed jointly at the Sternberg institute and the Institut de Mécanique Céleste et de Calcul des Ephémérides by Emelianov and Arlot. This database includes the widely used catalogue compiled by Morley (1989), which considers most of the ground-based observations from 1877 to 1982. Several other Earth-based observations (sometimes unpublished) are also included. Of great interest are the observations in 1988 by Kudryavtsev et al. (1992) at the time of the Phobos 2 mission.

Between 1988 and 1998, the Martian moons seem to have been disregarded by the observers. In 2003 Rohde, Ries, and Pascu (priv. com.) made 196 CCD observations over 4 nights of the Martian moons at Flagstaff. These are intersatellite observations (the observed position of Phobos with respect to the position of Deimos) and are among the most accurate observations made from the Earth.

The model presented here benefited from the large set of spacecraft observations done with Mariner 9, Viking 1-2, Phobos 2, but also from MGS (Bills et al. 2005) and Mars Express observations (Oberst et al. 2006). The last set includes 26 observations of Phobos with an accuracy of $0.5-5 \mathrm{~km}$ and 10 observations of Deimos with an accuracy of $1 \mathrm{~km}$.

Table 1 summarizes the whole set of observations considered in this work. A star (*) denotes unpublished observations. The letters $\mathrm{P}, \mathrm{D}$, and $\mathrm{M}$ denote respectively Phobos, Deimos, and Mars. $\mathrm{P} / \mathrm{M}$ and $\mathrm{D} / \mathrm{M}$ refers to observations relative to Mars, $\mathrm{P} / \mathrm{D}$ refers to intersatellite observations and $\mathrm{P}, \mathrm{D}$ are absolute observations. For the second and third columns respectively, the conventions are as follow: $1=$ true equator of the date, $2=$ B1900 equator, $3=$ B1950 equator, $4=$ J2000 equator; $0=$ separation and position angle coordinates $(s, p), 1=$ tangential coordinates $(X, Y), 2=$ differential coordinates $(\Delta \alpha, \Delta \delta), 3=$ absolute coordinates $(\alpha, \delta)$. A part of the data set has not been used after a preliminary study of the residuals revealed that there is a discrepancy between the different observational sets (see Sect. 5 for details).

In view of the number and full coverage of the most accurate observations of the Martian moons, we decided to perform three different fits. In particular, the possibility of using new spacecraft 
observations (MGS-MEX) makes it feasible to fit the Martian moons' ephemerides (and so the Martian dissipation) on shorter time scales than is usually done. The first fit introduced spacecraft observations from 1971 to 2005 only (Sect. 5.2). A second fit used the most accurate Earth-based astronomical observations starting with Pascu's observations in 1967 and all spacecraft observations until 2005. This fit is presented in Sect. 5.3. The last fit combined the spacecraft observations and a number of observations available from 1877 to 1950 . This fit is described in Sect. 5.4.

A limitation to the Viking observations (and to a lesser extent also the Mariner 9 observations) is the uncertainty in the spacecraft positions at the time of the observations. Those have been estimated to be $8 \mathrm{~km}$ and $4 \mathrm{~km}$ for Viking 1-2 and Mariner 9 spacecraft, respectively (Duxbury \& Callahan 1988, 1989). To reduce these uncertainties, the authors have suggested estimating two more parameters related to the spacecrafts' position errors, during the fit process. Following Chapront-Touze (1990), these have not been introduced to avoid a high number of parameters. We introduced updated spacecraft positions using SPICE kernel when available. These new positions were computed by Konopliv in 1995 (SPICE file header). We found differences between the SPICE positions and the positions published in Duxbury \& Callahan (1988), and Duxbury \& Callahan (1989) of less than $500 \mathrm{~m}$ for the Mariner 9 data and a few tens of kilometers for the Viking spacecraft.

\section{The numerical model}

The software used for numerical integration is called Numerical Orbit and Ephemerides (NOE) and is based on the work developed in Lainey et al. (2004). It was developed at the Royal Observatory of Belgium mainly for computing the ephemerides of natural satellites. It is an N-body code that incorporates highly sensitive modeling and can generate partial derivatives, which are needed to fit the initial positions, velocities, and other parameters (masses, $C_{20}, C_{40}, \ldots$ ) to the observation data.

The model presented in this work introduces (i) the Martian gravity field MGM1041C up to degree ${ }^{2} 10$ (Tyler et al. 2003); (ii) the perturbations of the Sun, Jupiter, Saturn, the Earth, and the Moon using DE406 ephemerides (Standish 1998); (iii) the mass of each Martian moon ${ }^{3}$ (Phobos mass borrowed from Tyler et al. 2003 and Deimos mass from Yuan et al. 2001); and (iv) the IAU2000 Martian precession/rotation (Seidelmann et al. 2002).

In principle, the secular acceleration (deceleration) of the Martian moons is directly related to the Martian Love number $k_{2}$, the Martian dissipation factor $Q$, and the mass of each moon. While the usual method fits two uncorrelated quadratic terms in the satellite longitudes, the tidal effects are modeled here in a more rigorous way by introducing a tidal potential. This method allows a better fit of the Phobos and Deimos secular accelerations by linking them to the same physical parameters. In particular, unrealistic acceleration of the motion of Deimos is automatically rejected, as Deimos revolves more slowly than Mars rotates. The tidal effects were introduced using Mignard's formulation (Mignard 1980), which models the tidal bulge by a time

${ }^{2}$ Former simulations introducing the Martian gravity field up to order 12 revealed no significant differences with respect to simulations introducing this potential only up to degree 10 .

${ }^{3}$ A preliminary study by Lainey \& Tanga (2005) in the context of the GAIA mission studied the small influence of the mutual perturbations of the Martian moons. In the present work, these forces have been introduced only for completeness. shift $\Delta t$ between the gravitational excitation by the satellite and the viscoelastic response of the planet. Actually $\Delta t$ can be related to the Martian dissipation factor $Q$ by the simple relation

$\Delta t=\frac{T \arcsin (1 / Q)}{2 \pi}$

where $T$ is the tidal excitation period depending on the Martian rotation period $T_{\mathrm{p}}$. The satellite orbital motion period $T_{\mathrm{s}}$ is related to it by

$T=\frac{T_{\mathrm{p}} T_{\mathrm{s}}}{2\left|T_{\mathrm{p}}-T_{\mathrm{s}}\right|}$

We introduced two different time shifts to take the tidal bulges of Phobos and Deimos into account. Mignard (1980) neglected the first part of his potential $U_{0}$, because he was mainly interested in the long-term evolution. This missing part has been added in the final tidal force $\boldsymbol{F}^{\mathrm{T}}$ acting on a moon, which is induced by the tidal bulge raised by this moon on the planet. $\boldsymbol{F}^{\mathrm{T}}$ takes the form

$$
\boldsymbol{F}^{\mathrm{T}}=-\frac{3 k_{2} G m^{2}\left(E_{r}\right)^{5}}{r^{8}}\left(\boldsymbol{r}+\Delta t\left[\frac{2 \boldsymbol{r}(\boldsymbol{r} \cdot \boldsymbol{v})}{r^{2}}+(\boldsymbol{r} \times \boldsymbol{\Omega}+\boldsymbol{v})\right]\right)
$$

where $m, E_{r}, \boldsymbol{r}, \boldsymbol{v}, \Omega$ are the moon mass, the Martian equatorial radius, the position and velocity vectors of the moon, and the Martian angular velocity vector, respectively. In the following, the Martian Love number has been fixed to $k_{2}=0.152$ (Konopliv et al. 2006). Bills et al. (2005) mention, however, the necessity of introducing the higher harmonic terms involving $k_{3}$ and $k_{4}$ in the dynamics. As these terms are still mostly unknown, it was preferred to keep only the $k_{2}$ term. Hence, the fit of the dissipation factor $Q$ more likely provides an effective value of $Q$ by partly absorbing the loss of the higher harmonics. The related acceleration in the model will, however, be correct.

The dynamical system is numerically integrated in a planetocentric frame with inertial axes (conveniently the Earth mean equator J2000). Hence, denoting $\boldsymbol{r}_{j}$ the position vector of a body $P_{j}$ (a satellite, the Sun, or a perturbing planet), the equation of motion of the satellites has the usual form of

$$
\begin{aligned}
\ddot{\boldsymbol{r}}_{i}= & -\frac{G\left(m_{0}+m_{i}\right) \boldsymbol{r}_{i}}{r_{i}^{3}}+\sum_{j=1, j \neq i}^{\mathcal{N}} G m_{j}\left(\frac{\boldsymbol{r}_{j}-\boldsymbol{r}_{i}}{r_{i j}^{3}}-\frac{\boldsymbol{r}_{j}}{r_{j}^{3}}\right) \\
& +G\left(m_{0}+m_{i}\right) \boldsymbol{\nabla}_{i} U_{\hat{\imath} \hat{0}}+\sum_{j=1, j \neq i}^{\mathcal{N}} G m_{j} \boldsymbol{\nabla}_{j} U_{\hat{\jmath} \hat{0}}
\end{aligned}
$$

where $U_{\hat{\jmath} \hat{0}}$ denotes the oblateness gravity field of the planet. The associated force is computed using a rotation matrix of angles ${ }^{4}$ $\left(\alpha_{0}+\frac{\pi}{2}, \frac{\pi}{2}-\delta_{0}, W\right)$ and its associated inverse.

Denoting $c_{l}$ as an unspecified parameter of the model that shall be fit (e.g. $\left.\boldsymbol{r}\left(t_{0}\right), \dot{\boldsymbol{r}}\left(t_{0}\right), Q \ldots\right)$, a useful relation is

$$
\frac{\partial}{\partial c_{l}}\left(\frac{\mathrm{d}^{2} \boldsymbol{r}_{i}}{\mathrm{~d} t^{2}}\right)=\frac{1}{m_{i}}\left[\sum_{j}\left(\frac{\partial \boldsymbol{F}_{i}}{\partial \boldsymbol{r}_{j}} \frac{\partial \boldsymbol{r}_{j}}{\partial c_{l}}+\frac{\partial \boldsymbol{F}_{i}}{\partial \dot{\boldsymbol{r}}_{j}} \frac{\partial \dot{\boldsymbol{r}}_{j}}{\partial c_{l}}\right)+\frac{\partial \boldsymbol{F}_{i}}{\partial c_{l}}\right]
$$

Hence, partial derivatives of the solutions with respect to initial positions and velocities of the satellites and dynamical parameters are computed from numerical integration of Eq. (4) and simultaneously with Eq. (5). For an explicit formulation of the dynamical equations and the variational equations used, we refer to Peters (1981) and Lainey et al. (2004).

A possible perturbation that may significantly decrease the accuracy of our model is the spin librations of the Martian

\footnotetext{
${ }^{4}$ The angles $\left(\alpha_{0}, \delta_{0}, W\right)$ are defined in Seidelmann et al. (2002).
} 

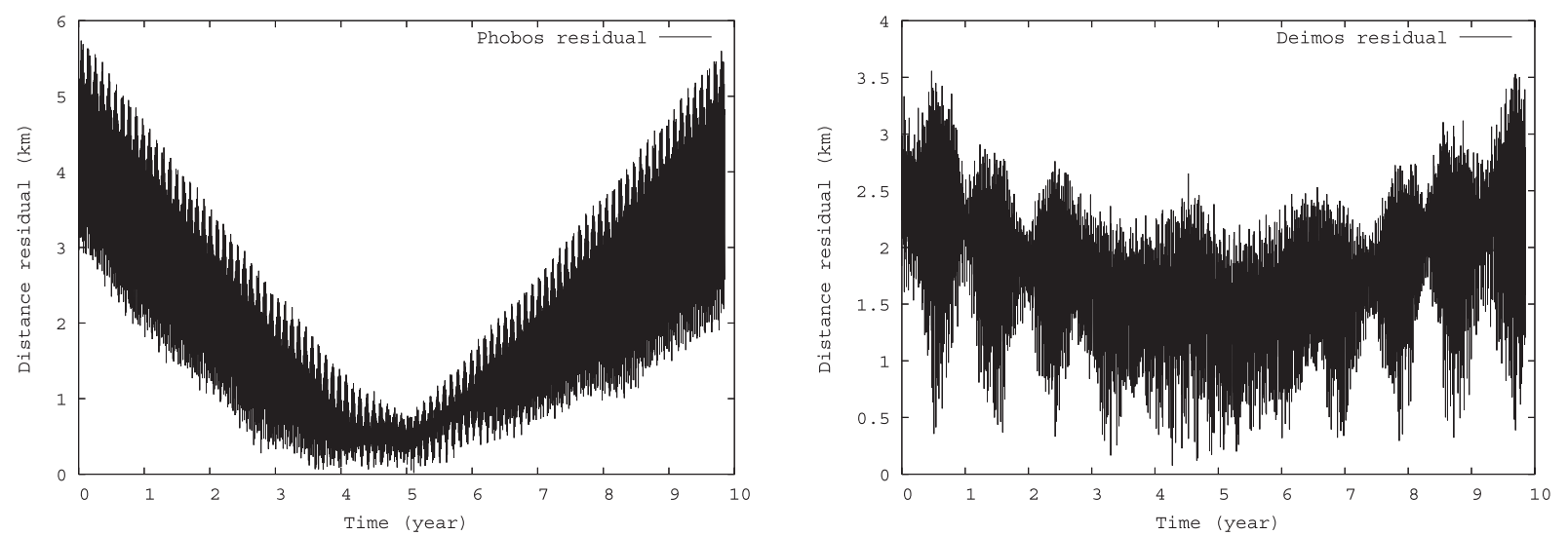

Fig. 1. Differences in distance after fitting the numerical model to the JPL ephemerides for Phobos (left panel) and Deimos (right panel). The satellite's initial positions and velocities and the Martian dissipation quality factor $Q$ have been fit here. The horizontal axes are in years relative to Julian day $2445053.5(25 / 03 / 1982)$.

moons. As both moons are in a spin-orbit resonance, induced librations affect the evolution of the satellite longitudes. So far, this perturbation has only been considered in the extensive work of Chapront-Touze (1990). However, the introduction of this resonance would need to consider the phase angle and its derivative at initial epoch as unknown parameters. As no observations of such an angle can be used easily, the fit of these parameters would be done on the basis of its influence on the orbital motion. Moreover, this sort of perturbation would require a good estimate of the $C_{20}$ parameter for both moons, which is not available, in particular after the still controversial value of the Phobos mass itself (Andert et al. 2004). Hence, the introduction of spin orbit librations in our model will be postponed to future work.

The integrator subroutine is from Everhart (1985) and called RA15. It was chosen for its computation speed and accuracy. During the different integrations, a constant step size of $\Delta t=$ 0.025 day was used. To increase the numerical accuracy during the fitting procedure (see Sect. 5), we performed forward and backward integrations starting at an initial Julian epoch of $2445053.5(25 / 03 / 1982 \mathrm{TDB})$. This epoch was chosen to keep high precision during the spacecraft era. The numerical accuracy of our simulation is at the level of a few tens of meters over 10 years and a few hundreds of meters over one century.

\section{Fit to JPL ephemerides}

To obtain good first estimates of the initial satellite positions and velocities, we fit the model to the JPL ephemerides of the Martian moons, which are based on the Jacobson et al. (1989) ephemerides. These computations were done over a time span of 3600 days with a rate of one value per day. The initial epoch was Julian day 2445053.5 (TDB time). The global modeling already described in Sect. 3 was considered. Differences in Cartesian positions for all satellites and the Martian dissipation factor $Q$ have been fit, with no weights assigned. The residuals after applying the least-square procedures are shown in Fig. 1. The resulting differences do not exceed a few kilometers and are distributed around a non-zero mean of the same order. In particular, the long-scale trends may be explained by the use of a different Martian gravity potential and precession rate. In addition, the assumed modeling precision of the JPL ephemerides is also one to two kilometers (Jacobson et al. 1989). This good agreement is sufficient to start a fitting procedure to the observations directly.

\section{Fit to the observations}

\subsection{The fitting procedure}

During the fitting procedure, time scale and light time corrections for each satellite-observer distance were introduced. The weights were computed by preliminary residuals, except for the spacecraft observations where the published weights were used. The only exception were the MOLA observations for which no weights are available. Hence, we used the published time offset given in Bills et al. (2005) for each shadow event and added an empirical uncertainty of $1.5 \mathrm{~s}$ to weight each event. All spacecraft observations were used, except the early Mariner 9 observations from the approach to Mars. The uncertainty in Mariner 9's position was about $50 \mathrm{~km}$ during this phase instead of only $4 \mathrm{~km}$ during the orbital phase (Duxbury \& Callahan 1989). These observations were used, however, for computing all rms post fit residuals to be in agreement with former published works. A three-sigma rejection criterion was used for Earth-based observations, but considering the low accuracy of early observations (pre-50 s observations) this criterion was reduced to two-sigma in this last case.

Earth-based observations involve just one satellite (absolute or relative positions to Mars) or intersatellite observations (Deimos relative to Phobos). In the first case, it is sometimes possible to deduce intersatellite positions when both satellites are present. We tried two possibilities, either always converting satellite positions as intersatellite positions or not, when fitting the data. Intersatellite positions increase the correlations between Phobos and Deimos initial state. In particular, observational error on one satellite can potentially be absorbed in changes in the orbital evolution of the second satellite. Intersatellite positions have not been considered in this paper when relative positions to Mars were available ${ }^{5}$.

The normal system was inverted by the least-square method (singular value decomposition). The fit parameters were the initial state vectors and the dissipation factor $Q$. After a few iterations, however, the solution quickly diverged. Actually, this problem was also encountered by Taylor (1998) with the Uranian satellites. He solved the problem by fitting initial elliptical

\footnotetext{
5 Intersatellite positions are often a powerful way to avoid the center of mass determination and ephemeris errors of the planet. It only makes sense if all satellites have the same magnitude (and so the same observational error). Moreover, the number of satellites must be high enough to avoid correlations.
} 
elements instead of initial Cartesian coordinates. As all former models were analytic so developed in elliptical elements, this problem has not occured with the Martian moons so far. More details concerning the fit of the elliptical elements can be found in Taylor (1998). An alternative way is to introduce constraints on the initial Cartesian coordinates to prevent high values that would induce a divergence in the solution. Both approaches using the same preliminary observational sets were tested. The data set was based on spacecraft observations only and covered 34 years. When fitting elliptical initial elements, the convergence was reached after only a few iterations. On the other hand, the constraint method was far less efficient and much more empirical. First, the magnitude of the uncertainties needed to be chosen carefully. A high value produced a diverging process, while a low value made the convergence very slow. After several trials, we finally found optimal uncertainties of $4 \times 10^{-9} \mathrm{AU}$ and $10^{-8} \mathrm{AU} /$ day for the position and velocity components, respectively. The constraint method needed around ten iterations where the former method needed only three or four. Therefore, only the elliptical fitting method was finally considered. Throughout the rest of this work, no constraints have been applied in the least-square process. All unknowns have been fit all together at each iteration, which includes the initial state vectors, the dissipation parameter $Q$, and the $C_{20}$ and $C_{22}$ coefficients of Phobos (see next section).

\subsection{Fit to spacecraft observations}

The spacecraft observations number in the hundreds and cover more than three decades. One should expect an accurate solution for both the Phobos and Deimos orbits using only those observations. The numerical model was fit to the spacecraft observations considering only the initial state vectors (by fit of the elliptical elements) of the two moons and the Martian dissipation factor $Q$ as unknown parameters. A first fit only used Mariner 9, Viking 1-2, and Phobos 2 observations. This fit was extended by introducing MOLA and SRC observations. Unfortunately, their introduction significantly increased the residuals of the former observations (essentially Mariner 9 and Viking). If the estimated accuracy of the spacecraft observations is correct (no biases in the position and the orientation of the spacecrafts), this effect indicates something lacking in the modelling of the perturbation. The most probable reason ${ }^{6}$ that may introduce this discrepancy would be the triaxiality of Phobos. In particular, the use of the actual known Martian gravity field (deduced only from spacecraft tracking) no longer introduces significant correlations with Phobos. The Phobos gravity coefficients $C_{20}$ and $C_{22}$ by Borderies \& Yoder (1990) were then used, which were computed from Phobos' topography and mass (assuming a constant density). After another trial, the residuals increased again by the same order of magnitude. It was thus decided to add Phobos' $C_{20}$ and $C_{22}$ coefficients as unknown parameters for the fit process. We found surprisingly very good residuals for all observations, with a consistent order of magnitude for the $C_{20}$ and $C_{22}$ values (without constraints). This result will be commented in Sect. 5.4 and in Sect. 8.

\subsection{Fit to modern ground-based observations}

Earth-based observations during the spacecraft era were added to the already considered spacecraft observations in the last run. Because discrepancies exist among the various observational

\footnotetext{
${ }^{6}$ Consideration of other perturbations will be in Sect. 8 .
}

sets, only the most accurate Earth-based observations were considered. These consist of 267 astrometrical observations done by Pascu at the Flagstaff and the Washington observatories between 1967 and 1971 (2 oppositions) and between 1982 and 1988 (4 oppositions). From the opposition of 1988, 813 intersatellite observations by Colas at Pic du Midi (Colas 1992), and 88 and 78 observations of Phobos and Deimos, respectively, done at La Palma by Jones et al. (1989), and 660 and 639 observations of Phobos and Deimos, respectively, done by Kudryavtsev et al. (1992) were included. Further 196 observations by Rohde, Ries, and Pascu at Flagstaff in 2003 were also added.

The post-fit residuals increased slightly compared to the former fit presented in Sect. 5.2. An explanation is that some observations by Pascu from 1982 to 1988 were probably biased, as found in the past by a comparison with other ephemeris sources (Pascu, priv. com.). These observations were reduced with regard to separation and position angle $(s, p)$, while the systematical bias probably affected only one of these two variables. Unfortunately, the software used in this work automatically converts $(s, p)$ variables into $(\Delta \alpha \cos \delta, \Delta \delta)$ coordinates, so no control of systematical errors could be done. As such a former correction had not been applied in the first step, this bias contaminates the results and compromises the quality of the fit. In the future, this treatment will be done in order to improve the final residuals. Despite this point, Pascu's observations are highly accurate and in addition provide satellite positions relative to Mars.

\subsection{Introducing the pre-50 s observations}

Earth-based observations made before 1950 were then introduced into the database. Although less accurate than modern observations, they cover a long time span before the spacecraft era (the oldest observations were made in 1877). For practical reasons, we did not introduce all observations, but only those that do not depend on the $(s, p)$ variables. The latter will be considered in a future work. Only very few iterations were required starting from the fit performed in Sect. 5.2, and the residuals did not change much. As this fit includes observations covering almost 130 years, this fit was chosen as the final solution for the ephemerides. Figure 2 shows Phobos and Deimos' post fit residuals from spacecraft observations. Figure 3 shows Phobos and Deimos from Earth-based observations (old and modern one). Tables 2 and 3 present the final rms post-fit residuals for each observational set. In particular, these tables can be compared to a similar table published in Chapront-Touze (1990). The MEX residuals clearly fit the expected accuracy of these observations. The MGS residuals are significantly larger, but a close look at Fig. 2 also shows periodic behavior. This may come from the use of former ephemerides models in the reduction process. Indeed, during the shadow-event reduction, only a time shift is fit. Hence, the satellite coordinates relative to Mars are computed from the JPL ephemerides. The other spacecraft residuals are the same order of magnitude as those published by Chapront-Touze (1990). Another method of comparison can directly compute the differences between both ephemerides. This is done in Sect. 7 with the JPL ephemerides.

The Martian dissipation factor $Q$ was found to be not very sensitive to the different sets of observations used. It was found to be equal to $79.61,78.70$, and 79.91 with similar error bars. The reference value chosen in this paper is $Q=79.91 \pm 0.69$, which derived from the last fit, assuming $k_{2}=0.152$ for the Martian Love number and $G m_{\mathrm{Ph}}=0.68012569 \times 10^{6} \mathrm{~m}^{3} / \mathrm{s}^{2}$ for the mass of Phobos. There is also a suspicion of a non 

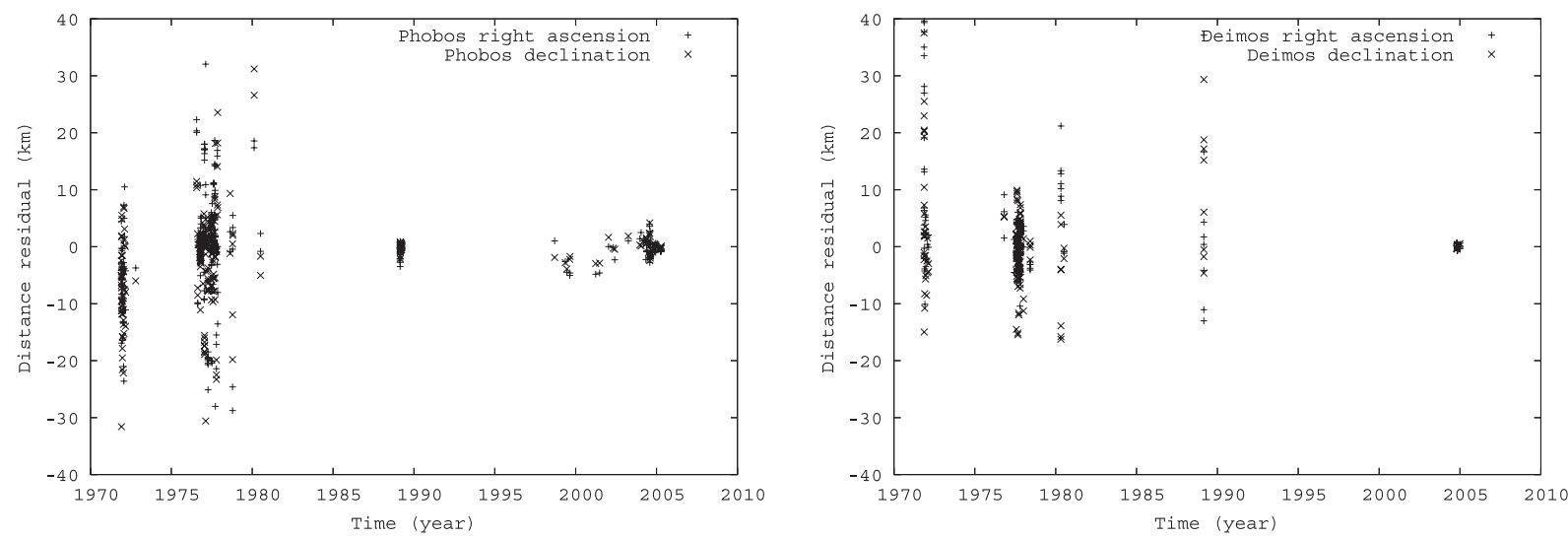

Fig. 2. Differences in distance after fit between the model and the spacecrafts observations for Phobos (left panel) and Deimos (right panel). The satellite initial positions, velocities and the Martian dissipation quality factor $Q$ and Phobos' $C_{20}$ and $C_{22}$ have been fit here.
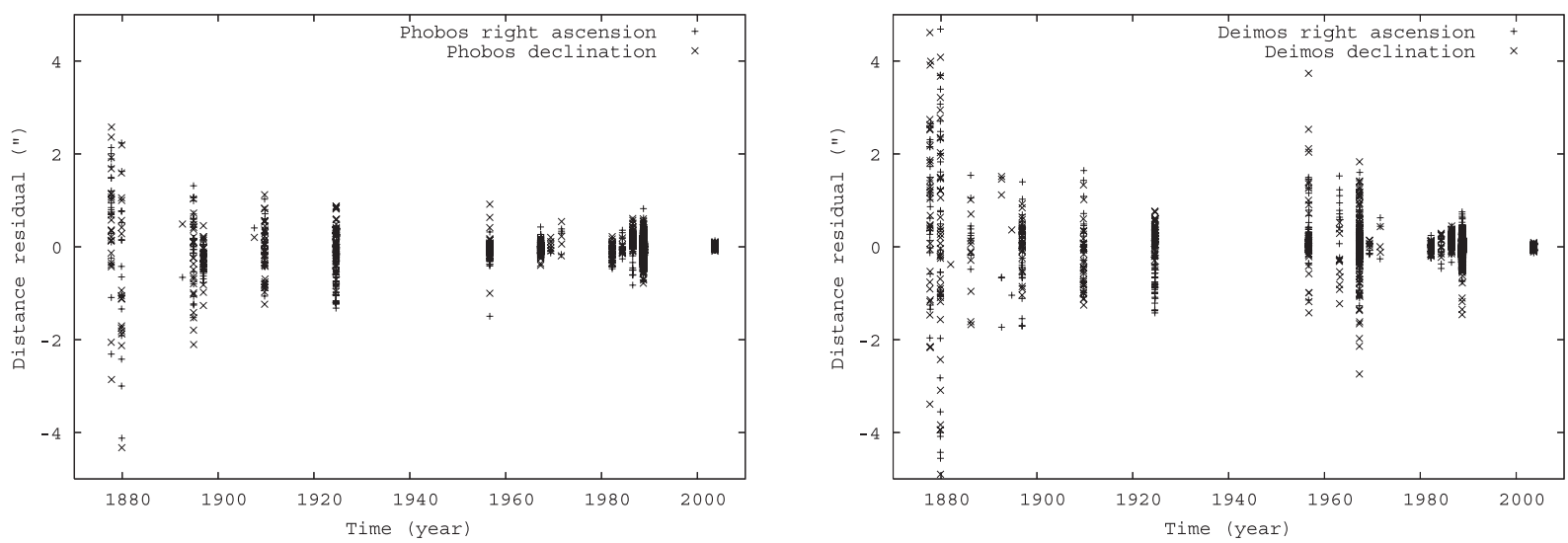

Fig. 3. Differences in distance after fit between the model and the ground observations for Phobos (left panel) and Deimos (right panel). The satellite initial positions, velocities and the Martian dissipation quality factor $Q$ and Phobos' $C_{20}$ and $C_{22}$ have been fit here.

Table 2. Mean $(v)$ and standard deviation $(\sigma)$ on right ascension and declination in seconds of degrees for each satellite.

\begin{tabular}{|c|c|c|c|c|c|c|}
\hline Observations & $\begin{array}{c}v_{\alpha} \\
\left({ }^{\prime \prime}\right)\end{array}$ & $\begin{array}{l}\sigma_{\alpha} \\
\left({ }^{\prime \prime}\right)\end{array}$ & $\begin{array}{r}v_{\delta} \\
\left({ }^{\prime \prime}\right)\end{array}$ & $\begin{array}{l}\sigma_{\delta} \\
\left({ }^{\prime \prime}\right)\end{array}$ & $N^{a}$ & satellite \\
\hline \multirow[t]{2}{*}{ Pascu (1995)* } & 0.00003 & 0.23234 & 0.04658 & 0.17103 & 217 & Phobos \\
\hline & 0.06439 & 0.20355 & 0.00943 & 0.22216 & 223 & Deimos \\
\hline \multirow{2}{*}{ Rohde, Ries, and Pascu (2003)* } & 0.00876 & 0.02901 & 0.00803 & 0.02826 & 196 & Phobos \\
\hline & -0.00876 & 0.02901 & -0.00803 & 0.02826 & 196 & Deimos \\
\hline \multirow[t]{2}{*}{ Colas (1992) } & -0.02147 & 0.05460 & -0.00226 & 0.06345 & 813 & Phobos \\
\hline & 0.02147 & 0.05460 & 0.00226 & 0.06345 & 813 & Deimos \\
\hline \multirow[t]{2}{*}{ Jones et al. (1989) } & -0.00213 & 0.11990 & 0.04983 & 0.11411 & 154 & Phobos \\
\hline & -0.06034 & 0.10228 & -0.02699 & 0.09801 & 78 & Deimos \\
\hline \multirow[t]{2}{*}{ Kudryavtsev et al. (1992) } & 0.09592 & 0.15067 & -0.12893 & 0.15058 & 660 & Phobos \\
\hline & 0.08342 & 0.15197 & -0.11304 & 0.14451 & 639 & Deimos \\
\hline Old Observations - Morley (1989) & -0.29783 & 4.01389 & -0.01730 & 1.60815 & 223 & Phobos \\
\hline Christie et al. (1878), Young (1880) & 0.00314 & 5.54741 & 0.35150 & 4.56567 & 340 & Deimos \\
\hline
\end{tabular}

* Unpublished observations.

${ }^{a}$ Number of observations by satellite.

homogeneous density for Phobos. The estimate of the satellite gravity field is $C_{20}=-0.072 \pm 0.013$ and $C_{22}=-0.048 \pm 0.002$. This clearly conflicts with a constant density assumption that would imply the values $C_{20}=-0.10058$ and $C_{22}=+0.01591$ (Borderies \& Yoder 1990). The negative sign of $C_{22}$, however, may suggest the signature of a remaining unmodeled perturbation, the use of a biased Martian gravity field, or the introduction of biased observations (see the discussion in Sect. 8).

The correlation of all the fit parameters are given in Table 4. The highest correlation is equal to 0.91 between $C_{20}$ and $C_{22}$.
These coefficients are not completely correlated, because the orbit of Phobos has a small inclination, and the orientation of its northern pole is assumed to be equal to the Martian one. For more details concerning the correlation of the $C_{20}$ and $C_{22}$ coefficients, one can refer to Jacobson \& Rush (2006) or Lainey (2002).

Table 5 presents a comparison between former Phobos' secular acceleration determinations. The result of this work is in good agreement with Jacobson et al. (1989) and Chapront-Touze (1990). The most recent determination by Bills et al. (2005) by 
Table 3. Mean $(v)$ and standard deviation $(\sigma)$ on right ascension and declination for each satellite.

\begin{tabular}{lrrrrrl}
\hline \hline Observations & $\begin{array}{r}v_{\alpha} \\
(\mathrm{km})\end{array}$ & $\begin{array}{r}\sigma_{\alpha} \\
(\mathrm{km})\end{array}$ & $\begin{array}{r}v_{\delta} \\
(\mathrm{km})\end{array}$ & $\begin{array}{r}\sigma_{\delta} \\
(\mathrm{km})\end{array}$ & $N$ & satellite \\
\hline MOLA (MGS) & -3.038 & 6.726 & 0.190 & 4.777 & 17 & Phobos \\
Bills et al. (2005) & - & - & - & - & - & Deimos \\
\hline Mariner 9 & -6.441 & 6.669 & -7.412 & 7.858 & 49 & Phobos \\
Duxbury \& Callahan (1989) & 15.080 & 19.992 & 5.008 & 13.677 & 31 & Deimos \\
\hline SRC (MEX) & 0.077 & 1.474 & 0.168 & 1.485 & 26 & Phobos \\
Oberst et al. (2006) & 0.002 & 0.517 & 0.044 & 0.322 & 10 & Deimos \\
\hline Viking 1-2 & 1.193 & 9.820 & -0.856 & 8.969 & 166 & Phobos \\
Duxbury \& Callahan (1988) & 0.477 & 4.443 & -2.433 & 11.539 & 109 & Deimos \\
\hline Phobos 2 & -0.350 & 0.967 & -0.032 & 0.594 & 37 & Phobos \\
Kolyuka et al. (1991) & 3.991 & 15.248 & 9.959 & 11.224 & 8 & Deimos \\
\hline
\end{tabular}

The angles have been multiplied by the distance between the point of observation and the two satellites to provide values in kilometers.

Table 4. Table of the correlations between all the fit parameters, including the initial elliptical elements, the dissipation factor $Q$, and the Phobos gravity coefficients $C_{20}$ and $C_{22}$.

\begin{tabular}{|c|c|c|c|c|c|c|c|c|c|c|c|c|c|c|c|}
\hline & $a_{1}$ & $L_{1}$ & $k_{1}$ & $h_{1}$ & $q_{1}$ & $p_{1}$ & $a_{2}$ & $L_{2}$ & $k_{2}$ & $h_{2}$ & $q_{2}$ & $p_{2}$ & $C_{20}$ & $C_{22}$ & $Q$ \\
\hline$a_{1}$ & 1.00 & 0.23 & 0.18 & 0.86 & 0.00 & -0.11 & 0.00 & 0.00 & 0.00 & 0.00 & 0.00 & 0.00 & 0.13 & 0.38 & 0.45 \\
\hline$L_{1}$ & 0.23 & 1.00 & 0.68 & 0.15 & -0.23 & 0.20 & 0.00 & 0.00 & 0.00 & 0.00 & 0.00 & 0.00 & 0.20 & 0.37 & 0.18 \\
\hline$k_{1}$ & 0.18 & 0.68 & 1.00 & 0.43 & -0.12 & -0.03 & 0.00 & 0.00 & 0.00 & 0.00 & 0.00 & 0.00 & 0.07 & 0.40 & -0.39 \\
\hline$h_{1}$ & 0.86 & 0.15 & 0.43 & 1.00 & 0.04 & -0.17 & 0.00 & 0.00 & 0.00 & 0.00 & 0.00 & 0.00 & 0.12 & 0.42 & 0.08 \\
\hline$q_{1}$ & 0.00 & -0.23 & -0.12 & 0.04 & 1.00 & -0.45 & 0.00 & 0.00 & 0.00 & 0.00 & 0.00 & 0.00 & -0.20 & -0.23 & 0.04 \\
\hline$p_{2}$ & -0.11 & 0.20 & -0.03 & -0.17 & -0.45 & 1.00 & 0.00 & 0.00 & 0.00 & 0.00 & 0.00 & 0.00 & 0.82 & 0.68 & -0.01 \\
\hline$a_{2}$ & 0.00 & 0.00 & 0.00 & 0.00 & 0.00 & 0.00 & 1.00 & 0.66 & -0.61 & -0.52 & 0.51 & 0.56 & 0.00 & 0.00 & 0.00 \\
\hline$k_{2}$ & 0.00 & 0.00 & 0.00 & 0.00 & 0.00 & 0.00 & -0.61 & -0.42 & 1.00 & -0.06 & 0.12 & 0.11 & 0.00 & 0.00 & 0.00 \\
\hline$h_{2}$ & 0.00 & 0.00 & 0.00 & 0.00 & 0.00 & 0.00 & -0.52 & -0.56 & -0.06 & 1.00 & -0.17 & -0.26 & 0.00 & 0.00 & 0.00 \\
\hline$q_{2}$ & 0.00 & 0.00 & 0.00 & 0.00 & 0.00 & 0.00 & 0.51 & 0.00 & 0.12 & -0.17 & 1.00 & 0.86 & 0.00 & 0.00 & 0.00 \\
\hline$p_{2}$ & 0.00 & 0.00 & 0.00 & 0.00 & 0.00 & 0.00 & 0.56 & 0.10 & 0.11 & -0.26 & 0.86 & 1.00 & 0.00 & 0.00 & 0.00 \\
\hline$C_{20}$ & 0.13 & 0.20 & 0.07 & 0.12 & -0.20 & 0.82 & 0.00 & 0.00 & 0.00 & 0.00 & 0.00 & 0.00 & 1.00 & 0.91 & 0.02 \\
\hline$C_{22}$ & 0.38 & 0.37 & 0.40 & 0.42 & -0.23 & 0.68 & 0.00 & 0.00 & 0.00 & 0.00 & 0.00 & 0.00 & 0.91 & 1.00 & -0.05 \\
\hline$Q$ & 0.45 & 0.18 & -0.39 & 0.08 & 0.04 & -0.01 & 0.00 & 0.00 & 0.00 & 0.00 & 0.00 & 0.00 & 0.02 & -0.05 & 1.00 \\
\hline
\end{tabular}

Elliptical elements have been taken under the regularized form $(a, L, k, h, q, p)$, where $a$ denotes the semi-major axis, $L$ denotes the mean longitude, $(k, h)$ are the real and imaginary component of $z=e \exp ^{\mathrm{i} \varpi}$ and $(q, p)$ are the real and imaginary component of $\zeta=\sin \frac{I}{2}$ exp $\mathrm{p}^{\mathrm{i} \Omega}$, where $\varpi$ is the longitude of the periapse, $e$ is the excentricity and $I$ is the inclination. Subscript 1 denotes the Phobos' elements and subscript 2 denotes the Deimos' elements. The correlations higher than 0.8 are shown in boldface.

Table 5. Phobos secular acceleration $\left(\frac{1}{2} \frac{\mathrm{d} n}{\mathrm{~d} t}\right)$ deduced from Martian moons ephemerides.

\begin{tabular}{lc}
\hline \hline source & $\begin{array}{c}\text { secular acceleration } \\
\frac{1}{2} \frac{\mathrm{d} n}{\mathrm{~d} t}\left(10^{-5} \mathrm{deg} / \mathrm{yr}^{2}\right)\end{array}$ \\
\hline Jacobson et al. (1989) & $124.9 \pm 1.8$ \\
Chapront-Touze (1990) & $127.0 \pm 0.8$ \\
Emelyanov et al. (1993) & $129.0 \pm 1.0$ \\
Bills et al. (2005) & $136.7 \pm 0.6$ \\
This work & $127.0 \pm 1.5$ \\
\hline
\end{tabular}

fitting MOLA observations is the most contrasting one. An explanation could be that in the work of Bills et al. (2005), only three terms arising in the expression of Phobos longitude were fit, and only MOLA observations used. Table 6 gives the initial state vectors at Julian epoch 2445053.5 computed as the initial state in the model.

\section{Signal processing and final representation}

The usual way to derive an ephemeris from numerical integration of a Solar system body in practical form is to fit Chebychev polynomials over the related numerical sampling. However, the difference between Chebychev polynomials and an analytical series (developed by means of analytical integration) is the size of the files involved. For slow objects like external satellites of Giant planets, Chebychev representations are quite convenient, but become inconvenient for fast satellites like Phobos. In order to avoid large volume files that may increase the computing time, we decided to perform a spectral analysis instead of fitting Chebychev polynomials. This will make our final ephemerides file smaller in size and easier to provide via the internet. Moreover, this will simplify further comparison between our ephemerides and the others, once each frequency is recognized. Last but not least, the frequency representation is defined far beyond the numerical integration time span.

The method and the software used for spectral analysis are very similar to the software presented in Vienne \& Duriez (1992) and based on the method presented in Laskar et al. (1992). The sampling step size was chosen after analyzing some small arcs at a very high step size. It appeared that no short periods with amplitudes higher than a few tens of meters were present for less than 0.1 day for Phobos and 0.4 day for Deimos. This allows use of a final step size of 0.05 and 0.2 day for Phobos and Deimos, respectively. To improve the algorithm, Chebychev polynomial (especially in the satellites longitude) were sometimes introduced. 
Table 6. Initial state vectors at the Julian epoch 2445053.5 (25/03/1982 TDB).

\begin{tabular}{lrrr}
\hline \hline Satellite & \multicolumn{1}{c}{$x$} & \multicolumn{1}{c}{$y$} & \multicolumn{1}{c}{$z$} \\
\hline position of Phobos (AU) & $-5.275254348561059 \mathrm{E}-005$ & $4.940685958944592 \mathrm{E}-006$ & $3.208377735462846 \mathrm{E}-005$ \\
velocity of Phobos (AU/day) & $-3.419274688341861 \mathrm{E}-004$ & $-1.145431373878196 \mathrm{E}-003$ & $-3.624022104187871 \mathrm{E}-004$ \\
position of Deimos (AU) & $5.339886204059241 \mathrm{E}-005$ & $-1.211337469486434 \mathrm{E}-004$ & $-8.411522628553895 \mathrm{E}-005$ \\
velocity of Deimos (AU/day) & $6.576371605012466 \mathrm{E}-004$ & $3.927174026111411 \mathrm{E}-004$ & $-1.484537310476662 \mathrm{E}-004$ \\
\hline
\end{tabular}
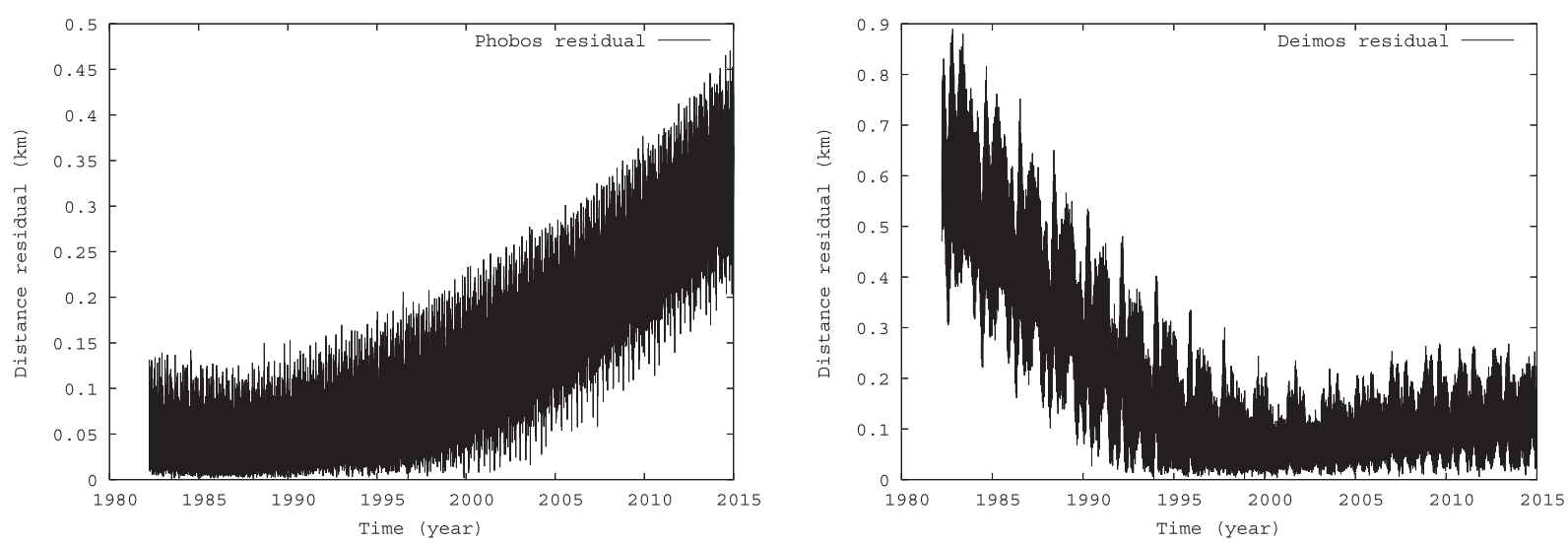

Fig. 4. The internal precision of the ephemerides reflects the differences between the numerical integration and the frequency reconstruction for Phobos (left panel) and Deimos (right panel).

Figure 4 shows the differences between our final frequency representation and the initial sampled time series produced by numerical integration. The differences reveal the internal precision of the final ephemerides, which is a few hundred meters during the period 1990-2015. The frequency analysis may be improved in the future when new spacecraft observations are available.

\section{Comparison with JPL and ESOC ephemerides}

Finally, the ephemerides were compared with the JPL and ESOC ephemerides. Both are available as SPICE kernels and defined over the time span 1976-2025 and 2004-2006, respectively. Figure 5 presents the related differences, which agree with the results found by Bills et al. (2005), Bell et al. (2005), and Oberst et al. (2006) using the MOLA, MER, and SRC instruments. In particular, JPL ephemerides are drifting compared to the ephemerides presented here, while ESOC's ephemerides show a higher but periodic scattering and a shift of $9 \mathrm{~km}$ and $15 \mathrm{~km}$ with respect to Phobos and Deimos. It shall be noted that the ephemerides from the model of this paper agree with the JPL ephemerides during the Viking and Phobos 2 era. This leads to the conclusion that the spacecrafts residuals shown in Table 3 are mainly observational errors.

\section{Consideration of non introduced perturbations}

As explained in Sect. 1, the introduction of the Phobos' $C_{20}$ and $C_{22}$ gravity field coefficients mainly induce some linear drifts in the angular elements (Borderies \& Yoder 1990). An easy way to quantify them is to perform the difference between a first simulation involving the $C_{20}$ and $C_{22}$ coefficients, and a second simulation without them. This difference was performed over 3 years, and is presented in Fig. 6. The initial conditions and forces involved in the physical modeling are exactly the same as the ones used in the last fit of the present ephemerides. The Phobos' $C_{20}$ and $C_{22}$ coefficients introduce a secular drift of roughly $200 \mathrm{~km}$ over 3 years. A possible explanation for the negative (and so unphysical) sign of $C_{22}$ could be that a perturbation at the same order of magnitude has been neglected in the present model. To verify such an eventuality, three perturbations not introduced in the former force model were checked. The differences between one simulation with and one simulation without each perturbation tested are presented in Fig. 6. The first perturbation tested is the influence of the temporal variation of the Martian $C_{20}$ coefficient. The numerical values for the expresssion of the temporal signature were taken from Konopliv et al. (2006). The simulations indicate that this perturbation introduces some periodic terms with an amplitude of only a few tens of meters. The second perturbation that has been tested is the presence of the Solar tidal bulge raised on Mars. The main related effect is a secular trend of $60 \mathrm{~m}$ over three years. The last pertubation tested was the Martian nutation missing in the present model. As the Martian nutation is unobserved so far, the presumed nutation was taken from the numerical model of the Martian rotation provided by Rambaux (priv. com.). The numerical model called SONYR was applied to Mercury (Rambaux \& Bois 2004). The global effect of the nutation is a secular trend of $300 \mathrm{~m}$ over 3 years on the Phobos' longitude.

It appears that none of the three perturbations considered in this section reaches the order of magnitude of the perturbation associated with Phobos' $C_{20}$ and $C_{22}$ coefficients. Although some other perturbations have not been tested (Phobos' libration, radiation pressure, and Yarkovsky effect thought to be small), it seems, however, more likely that the Martian gravity field used or some of the spacecraft observations are biased. More importantly, it has been shown that Phobos' oblateness gravity field contributes at an observable level to the Martian moon dynamics and could be, in principle, fit from astrometric observations.

\section{Conclusion}

New ephemerides of the Martian moons have been developed on the basis of MEX, MGS, Phobos 2, Viking 1-2, Mariner 9, and ground based observations. The recent observed differences 

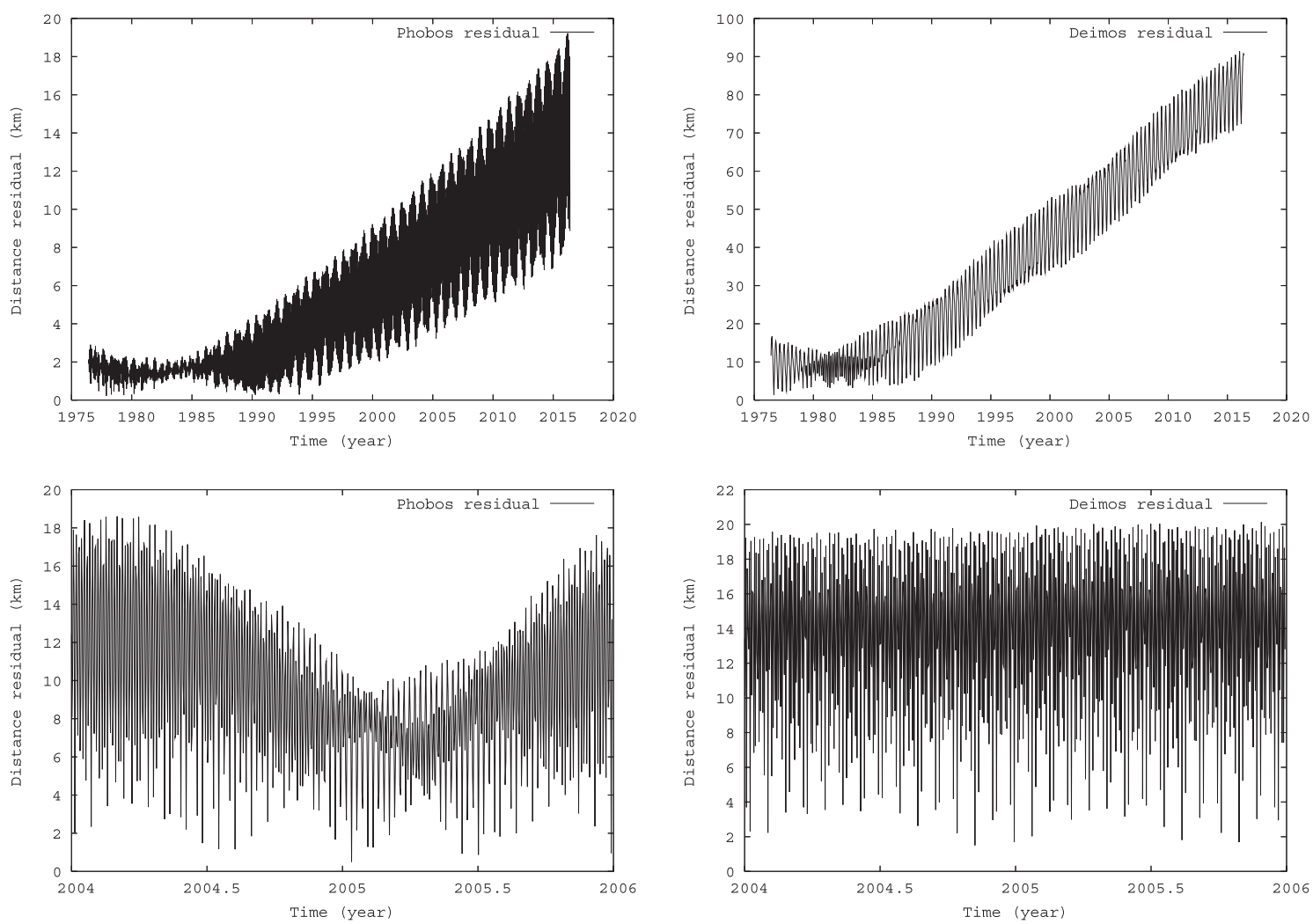

Fig. 5. Comparison between the computed ephemerides and ephemerides from other sources. The graphs present the differences in distance between the position of Phobos (left) and Deimos (right) using the model with respect to the JPL (top) model and the ESOC (bottom) model.
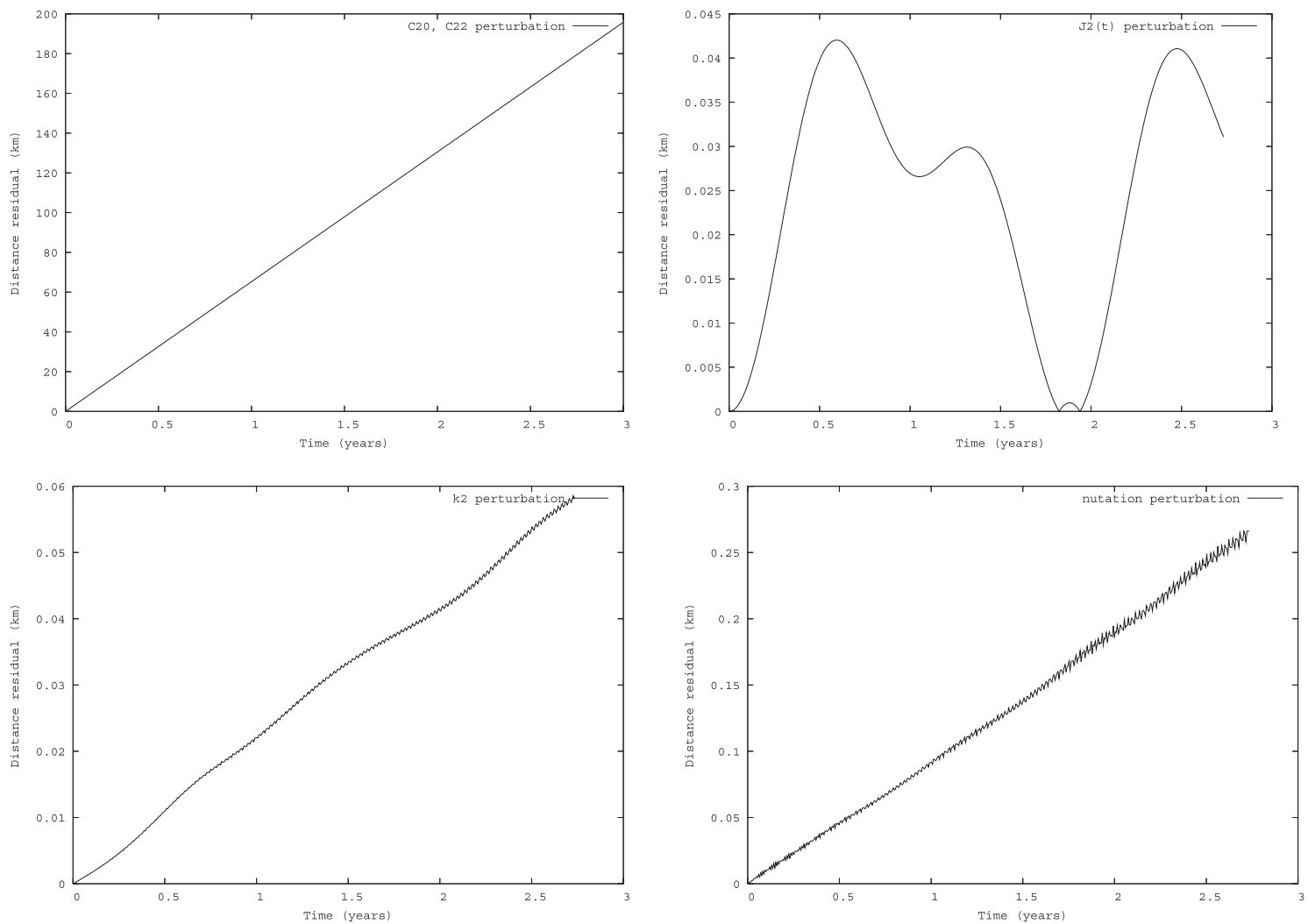

Fig. 6. Differences between one numerical simulation introducing the force modeling of reference in this paper and other simulations adding one perturbation to be tested. The perturbations tested are the Phobos' $C_{20}$ and $C_{22}$ coefficients (upper left panel), the temporal variation of the Martian $C_{20}$ coefficient (upper right panel), the Solar tidal bulge raised on Mars (lower left panel), and the Martian nutation (lower right panel). 
of up to ten kilometers between the prediction and the true satellite locations observed by MGS and MEX do not appear in this solution. Two different fitting methods were tested during the adjustment of the model to the observations. The use of elliptical elements during the fit process is by far more efficient than fitting Cartesian coordinates (even introducing constraints). This is also the first completely numerical ephemerides of Phobos and Deimos. The physical formulation of the tidal effects of the Mars/moon systems has been successfully introduced. The high Martian internal dissipation quantified at the time of Phobos 2 mission is confirmed. This dissipation factor is found to be $Q=79.91 \pm 0.69$, assuming $k_{2}=0.152$ for the Martian Love number and $G m_{\mathrm{Ph}}=0.68 \times 10^{6} \mathrm{~m}^{3} / \mathrm{s}^{2}$ for the Phobos mass. The uncertainties of the last two values constrain the determination of $Q$. Unexpectedly, there is suspicion of a non-uniform density of Phobos. The solution for the Phobos gravity field is indeed found to be $C_{20}=-0.0719 \pm 0.013$ and $C_{22}=-0.0481 \pm 0.002$ and is different from a gravity field derived by a shape model assuming uniform density. If the solution in the present paper is not completely satisfying, at least it demonstrates the sensitivity of the Martian moon ephemerides to Phobos' gravity field.

The Mars Express mission has been extended for two more years and will provide many more precise astrometric observations of the Martian satellites. The present model will be regularly updated by introducing these new observations, as well as new MOLA observations (Bills, personnal communication) in the future.

The Phobos libration has not been included in the model so far. It is a perturbation that will be introduced in further improvements. This will affect the previous determination of the Phobos gravity coefficients somewhat and will further improve the ephemerides residuals.

The accuracy of the computed ephemerides is expected to be roughly one kilometer over the presented period. A FORTRAN subroutine computing the Martian moons ephemerides is available on request.

While writing this paper, new JPL ephemerides have been released (Jacobson \& Rush 2006). A comparison done there demonstrates a good agreement between our ephemerides and the new JPL ones.

Acknowledgements. The authors are grateful to D. Pascu for providing them all of his already reduced observations done at USNO and to J. Oberst for providing the SRC observations (Mars Express) of the Martian moons. The authors would also like to thank F. Lemoine for directing our attention to the possibility of improving the Mariner 9 and Viking 1-2 spacecraft positions. Our thanks go to S. Le Maistre and G. Pfyffer for computing the SPICE kernel. The authors are grateful to P. Rosenblatt, T. Van Hoolst, and N. Emelianov for fruitful discussions.

This work benefited from the support of the European Community's Improving Human Potential Programme under contract RTN2-2001-00414, MAGE. M.P. acknowledges support from BMFT via DLR under grant 50QP9909.

\section{References}

Andert, T. P., Pätzold, M., Lainey, V., et al. 2004, AGU Fall Meeting Abstracts, A802
Bell, J. F., Lemmon, M. T., Duxbury, T. C., et al. 2005, Nature, 436, 55 Bills, B. G., Neumann, G. A., Smith, D. E., \& Zuber, M. T. 2005, J. Geophys. Res. (Planets), 110, 7004

Bobylev, V. V., Evdokimov, A. E., Kalinichenko, O. A., et al. 1991, Izvestiya Glavnoj Astronomicheskoj Observatorii v Pulkove, 207, 37

Borderies, N., \& Yoder, C. F. 1990, A\&A, 233, 235

Chapront-Touze, M. 1990, A\&A, 240, 159

Christie, W., Erck, W., \& Pritchard, C. 1878, MNRAS, 38, 361

Colas, F. 1992, A\&AS, 96, 485

Duxbury, T., \& Callahan, J. D. 1988, A\&A, 201, 169

Duxbury, T. C., \& Callahan, J. D. 1989, A\&A, 216, 284

Emelyanov, N. V., Vashkovyak, S. N., \& Nasonova, L. P. 1993, A\&A, 267, 634

Everhart, E. 1985, in Dynamics of Comets: Their Origin and Evolution, ASSL 115: IAU Colloq. 83, 185

Izhakevich, R., Kaltygina, S., Ledovskaya, I. V., Sereda, E., \& Shatokhina, S. 1990, Scientific paper deposited in All-russian institute of scientific and technical information, 1

Jacobson, R., \& Rush, B. 2006, JPL IOM, 225, 1

Jacobson, R., Synnott, S. P., \& Campbell, J. K. C. 1989, A\&A, 225, 548

Jones, D. H. P., Sinclair, A. T., \& Williams, I. P. 1989, MNRAS, 237, 15P

Kalinichenko, O. A., Kiseleva, T. P., Salukvadze, G. N., \& Chanturiia, S. M. 1990, Abastumanskaia Astrofizicheskaia Observatoriia Biulleten, 68, 99

Kiselev, A. A., Kiseleva, T. P., Bobylev, V. V., et al. 1989, Izvestiya Glavnoj Astronomicheskoj Observatorii v Pulkove, 206, 33

Kiseleva, T. P., \& Chanturiia, S. M. 1988, Abastumanskaia Astrofizicheskaia Observatoriia Biulleten, 63, 31

Kolyuka, Y., Tikhonov, V., Ivanov, N., et al. 1991, A\&A, 244, 236

Konopliv, A. S., Yoder, C. F., Standish, E. M., Yuan, D., \& Sjogren, W. L. 2006, Icarus, 182, 23

Kudryavtsev, S. M., Shokin, Y. A., \& Yevstigneeva, N. M. 1992, Preprint. Sternberg State Astronomical Institute, 24, 1

Lainey, V. 2002, Thèse de l'Observatoire de Paris, 1

Lainey, V., \& Tanga, P. 2005, AAS/Division for Planetary Sciences Meeting Abstracts, 37

Lainey, V., Duriez, L., \& Vienne, A. 2004, A\&A, 420, 1171

Laskar, J., Froeschlé, C., \& Celletti, A. 1992, Physica D Nonlinear Phenomena, 56,253

Lemoine, F. G., Smith, D. E., Rowlands, D. D., et al. 2001, J. Geophys. Res., 106, 23359

Mignard, F. 1980, Moon and Planets, 23, 185

Morley, T. 1989, A\&AS, 77, 209

Morley, T. A. 1990, A\&A, 228, 260

Neumann, G. A., Bills, B. G., Smith, D. E., \& Zuber, M. T. 2004, in Lunar and Planetary Institute Conference Abstracts, 1820

Nikonov, O. V., Zhilinskii, E. G., Bobylev, V. V., \& Nikonova, E. S. 1991, Izvestiya Glavnoj Astronomicheskoj Observatorii v Pulkove, 207, 52

Oberst, J., Matz, K., Roatsch, T., et al. 2006, A\&A, 447, 1145

Peters, C. F. 1981, A\&A, 104, 37

Rambaux, N., \& Bois, E. 2004, A\&A, 413, 381

Seidelmann, P. K., Abalakin, V. K., Bursa, M., et al. 2002, Celestial Mechanics and Dynamical Astronomy, 82, 83

Sharpless, B. P. 1945, AJ, 51, 185

Shor, V. 1989, Preprint of Sternberg Astronomical Institute, 13

Shor, V. A. 1975, Celestial Mechanics, 12, 61

Sinclair, A. 1978, Vistas Astron., 22, 133

Sinclair, A. T. 1972, MNRAS, 155, 249

Standish, E. 1998, JPL IOM

Taylor, D. B. 1998, A\&A, 330, 362

Tel'nyuk-Adamchuk, V., Churyumov, K. I., Pikalo, N., et al. 1990, Manuscript deponent at the Ukrainean science and technical information institute, 1

Tyler, G., Balmino, G., Hinson, D., et al. 2003, USA_NASA JPL_MORS 1021

Vienne, A., \& Duriez, L. 1992, A\&A, 257, 331

Young, C. A. 1880, The Observatory, 3, 270

Yuan, D.-N., Sjogren, W. L., Konopliv, A. S., \& Kucinskas, A. B. 2001, J. Geophys. Res., 106, 23377 